\title{
Algunas reflexiones acerca de los "maestros de hacer breviarios y escrituras de molde" Juan de Bobadilla y Álvaro de Castro (1483)
}

\author{
Benito RIAL COSTAS \\ benito@ombrettaetc.net
}

Recibido: mayo 2011

Aceptado: octubre 2011

Resumen: En 1483 el Cabildo compostelano acuerda con los "maestros de hacer breviarios y escrituras de molde" Juan de Bobadilla y Álvaro de Castro la impresión de ciento veinte breviarios para la diócesis compostelana. Pocos años después del descubrimiento de este documento, a finales del siglo XIX, la historiografía creó unas biografías de Juan de Bobadilla y Álvaro de Castro que hoy son comúnmente aceptadas.

Este artículo repasa la historia de la creación de dichas biografías, exponiendo la poca solidez de los argumentos utilizados; y propone limitarse a los datos de Juan de Bobadilla y Álvaro de Castro que se mencionan en el acuerdo de impresión de 1483, cuestionando, sin embargo, la interpretación que la historiografía ha hecho de ese documento.

Palabras clave: Juan de Bobadilla; Álvaro de Castro; Santiago de Compostela; Huete; Breviario compostelano; Ordenanzas Reales; Antonio de Centenera; Burgos; Villasandino, Toledo.

\section{Some Considerations On the 'maestros de hacer breviarios y escrituras de molde' Juan de Bobadilla and Álvaro de Castro (1483)}

\begin{abstract}
In 1483, the Archdiocesan Council of Santiago agreed with the 'maestros de hacer breviarios y escrituras de molde' Juan de Bobadilla and Álvaro de Castro on printing one hundred twenty copies of a Compostelan breviary. A few years after this document's discovery, at the end of nineteenth century, historiography created Juan de Bobadilla and Álvaro de Castro's biographies, which are still today largely accepted.

This article reviews the history of this biographic creation, exposing the weaknesses of the arguments that have been used in their ongoing reproduction. The article proposes that Juan de Bobadilla and Álvaro de Castro's biographic data should be limited to those contained in the 1483 's agreement. It questions, however, the interpretation that historians have made of this document.
\end{abstract}

Keywords: Juan de Bobadilla; Álvaro de Castro; St. James of Compostela; Huete; Compostelan Breviary; Ordenanzas Reales; Antonio de Centenera; Burgos; Villasandino, Toledo. 


\section{INTRODUCCIÓN}

En 1883 el canónigo de la catedral compostelana y miembro de la Real Academia de la Historia, Antonio López Ferreiro, daba a conocer el primer acuerdo de impresión del que se tiene noticia en Galicia. El 17 de junio de 1483 el cabildo compostelano acordaba con los "maestros de hacer breviarios y escrituras de molde" Juan de Bobadilla, vecino de Burgos, y Álvaro de Castro, vecino de Villasandino, la impresión en papel de ciento veinte breviarios para la diócesis compostelana. Dicho acuerdo indicaba la posible realización de un breviario compostelano entre 1483 y 1484 del que hasta ese momento nada se sabía, y daba a conocer dos nuevos nombres para la historia de la imprenta en Castilla:

$\mathrm{F}[\mathrm{ec}] \mathrm{ha}$ xvii días do mes de junio anno de lxxxiii [...]. Los sobre dichos señores [del cabildo compostelano] obligaron a si e los bienes de la mesa capitular de tomar cento e vinte breuiarios a rrespeto de treinta e çinco rreales cada briuiario a Ju[an] d[e] Bobadilla, vezino d[e] Burgos, e a Álu[ar]o de Castro, vezino de Villasendino, maestros de faser briuiarios e escripturas de moldes. Los q[ua]les çie[n]to e vynte briuiarios de moldes han de ser de buena letra segund[o] paresçió por hun dos cardernos q[ue] dexaron de mostra. Los quales d[ic]hos briuiarios han de faser e traer fechos a esta çibdad del día de sant Jua[n] p[ri]mero q[ue] biene en adelante fasta hun año, e se antes vinieren $\mathrm{q}[\mathrm{ue}]$ los $\mathrm{d}[\mathrm{ic}]$ hos bribiarios los rreçiban e se más briuiarios traxieren $\mathrm{d}[\mathrm{e}]$ los sobre $\mathrm{d}[\mathrm{ic}]$ hos çiento e veinte $\mathrm{q}[\mathrm{ue}]$ los no puedan vender fasta $\mathrm{q}[\mathrm{ue}]$ los çiento e vinte del $\mathrm{d}[\mathrm{ic}]$ ho cabildo sean destribuydos. Los quales $\mathrm{d}[\mathrm{ic}]$ hos çiento e vinte briuiarios han de ser por la costumbre comp[ostela]na, escriptos en papel e aluminados e encadernados. E del día $\mathrm{q}[\mathrm{ue}]$ binieren en adelante fasta vn mes el cab[ild]o le ha de pagar la mestad de los d[ic]hos briuiarios e la otra mestad dando a otro mes. ${ }^{1}$

El encargo de ciento veinte breviarios a dos completos desconocidos en la historia de la imprenta y la ausencia total de ejemplares de dicho breviario o noticias acerca de su entrega, plantearon inmediatamente la necesidad de confirmar su efectiva realización, descubrir el lugar de su impresión, y conocer la identidad y verdadera labor de los dos "maestros de hacer breviarios" que se mencionaban en dicho acuerdo.

1 Archivo de la Catedral de Santiago. Actas Capitulares. Libro 2 ${ }^{\circ}$ 1481-1591. Legajo 476, folio 19 verso. Cfr. López Ferreiro, 1883 (1968): 231. 


\section{JUAN DE BOBADILLA}

Después de más de cien años del descubrimiento de López Ferreiro, la única información que se posee de Juan de Bobadilla es la que se nos ofrece en este encargo de impresión; un escueto "vecino de Burgos" y, junto con su compañero, maestro de hacer breviarios y escrituras de molde, que ha llevado a todos los investigadores sin excepción, desde Konrad Haebler a Antonio Odriozola, pasando por María Luisa Cuesta, Antonio Rey Soto y Atanasio López, a señalar únicamente su vecindad burgalesa y su oficio de impresor. ${ }^{2}$ Todos los esfuerzos realizados para completar esta lacónica biografía han resultado infructuosos.

La posibilidad que Konrad Haebler apuntaba en 1917 de que fuese obra de Juan de Bobadilla un Flos Sanctorum sin pie de imprenta citado tiempo atrás por José Gallardo, fue años más tarde totalmente descartada por Francisco Vindel. Hoy es comunmente aceptada la falta de relación entre el dicho Flos Sanctorum y el Juan de Bobadilla que se presentaba ante el cabildo compostelano en $1483 .{ }^{3}$

Es producción [el Flos Sanctorum] de un taller de que hasta el día no se conoce ninguna otra obra, pero el impresor no fué un principiante sino un maestro acabado en el arte, que supo disponer muy bien sus materiales, supo fundir letras muy buenas de formas inusitadas pero bellas, no toscas, y supo grabar capitales bastante bonitas. $\mathrm{Su}$ letrería, aunque de formas originales, se parece algún tanto a la de Alfonso(sic) de Castro, el de Huete, y por eso emito con todas las precauciones debidas la hipótesis, de que tal vez el compañero que tuvo Castro en Santiago, Juan de Bobadilla, pueda ser el que imprimió el libro. Pero para esta suposición no hay fundamento exterior alguno, y tan solo es fruto de meras conjeturas. ${ }^{4}$

No comprendemos cómo el Dr. Haebler pudo emitir juicio tan absurdo sobre la tipografia de este libro [...].

La tipografía de este libro [el Flos Sanctorum] no puede ser más arcaica, ni su estudio deja de comprobar que se trata de uno de los más primitivos trabajos que se conocen de la Imprenta en España. ${ }^{5}$

2 Haebler, 1903 (1992): 36-37; Odriozola, 1987; Odriozola y Barreiro, 1992: 47; Cuesta, 1932: 203; Rey Soto, 1934 (1988): 20-24; y López, 1935: 102-103.

3 Gallardo, 1863-1889: I, 814-815; y Vindel, 1945-1954: VIII, xiv-xv. Véase también Incunabula Short Title Catalogue <http://istc.bl.uk/search/search.html?operation=record\&rsid $=292392 \& \mathrm{q}=0>$ [consultado el 25 de enero de 2010].

4 Haebler, 1917 (1992): 76 . Véase también Rey Soto, 1934 (1988): 20-24; y López, 1935: 102-103.

5 Vindel, 1945-1954: VIII, xiv. 
Ningún otro documento en el archivo de la catedral compostelana, ni ninguna otra noticia en la historia de la imprenta, hacen referencia a tal impresor. Ni siquiera el reciente trabajo de catalogación de los fondos documentales del Archivo Histórico de la Catedral de Burgos ha aportado algún otro dato acerca de él. ${ }^{6}$

\section{3 ÁLVARO DE CASTRO}

La historia de Álvaro de Castro, en cambio, ha corrido una suerte muy difierente. Antonio López Ferreiro en 1883 se limitaba a recoger los datos registrados en el acuerdo de impresión de los ciento veinte breviarios compostelanos, señalando un nombre - Álvaro de Castro-, un lugar -Villasandino- y un oficio -maestro de hacer breviarios y escrituras de molde. ${ }^{7}$ Pero en 1903 Konrad Haebler, en esta ocasión con mucha más fortuna que con Juan de Bobadilla, completará estos datos, identificándo al Álvaro de Castro de 1483 con el impresor "Castro" que parece trabajar en Huete en torno a esos mismos años. ${ }^{8}$

Detengámonos por un momento en la figura del impresor "Castro" de Huete pues es importante para entender lo que expondremos más adelante, ya que su existencia no estuvo exenta de polémica y su labor de impresor no fue siempre aceptada, ni siquiera por parte del propio Haebler.

\section{1. "CASTRO" Y LAS ORDENANZAS REALES DE DÍAZ DE MON- TALVO}

Konrad Haebler, para intentar demostrar la presencia en Huete durante las dos últimas décadas del siglo XV de un impresor llamado "Castro", se basaba en 1903 en los colofones de dos impresiones de las Ordenanzas Reales de Díaz de Montalvo; una fechada en Huete el 11 de noviembre de 1484 y la otra, siempre en Huete, el 23 de agosto de 1485:

Por mandado de los muy al $\mid$ tos y muy poderosos sere $\mid$ nisimos y cristianisimos $\mid$ principes Rey don Fernan | do y Reina doña Isabel nuestros se | ñores compuso este libro de leyes el Doc | tor Alfonso Diaz de Montalvo oidor de su | audiencia y su refrendario y de su | Consejo y acabose de escrivir en la cibdad | de Huepte a once dias del mes de no | viembre dia de San Martin año del nascimiento | del nues-

6 Informatización y catalogación $<$ http://www.cajacirculo.com/es/index.php?PA_ID=476> [consultado el 3 de noviembre de 2009].

7 López Ferreiro, 1883 (1968): 231; y Cuesta, 1932: 203. María Luisa Cuesta llama por error a Álvaro de Castro "Alonso de Castro", pasa por alto la identificación de Haebler con el "Castro" de Huete y menciona tan solo las noticias dadas por López Ferreiro.

8 Haebler, 1903 (1992): 98-100. 
tro salvador | Jesucristo de mil y qua | trocientos y ochenta y quatro años $\mid$ Castro. ${ }^{9}$

Por mandado de los muy $\mid$ altos y muy poderosos | serenisimos y cristiani | simos principes Rey don Fernan | do y Reina doña Isabel nuestros seño | res compuso este libro de leyes el | Doctor Alfonso Diaz de Montalvo | oidor de su Audiencia e su re | frendario e de su Consejo y aca $\mid$ bose de escrevir en la cibdad de | Huepte a veinte y tres del mes $\mid$ de agosto vispera de señor Sant | Bartolome año del nascimiento $\mid$ de nuestro salvador Jesucristo de mill | y cuatrocientos y ochenta y cinco años $\mid$ Castro. $^{10}$

Dado lo poco explícito de los colofones y hasta que se descubrió el segundo de estos dos textos impresos, la crítica en general tendía a no interpretar "Castro" como el nombre del impresor de las Ordenanzas. Basándose en el colofón del 11 de noviembre de 1484, Dionisio Hidalgo en 1861 suponía a "Castro" el amanuense encargado de transcribir el texto y adjudicaba su impresión a Antonio de Centenera en Zamora. ${ }^{11}$ Fermín Caballero en 1873 creía todavía difícil que hubiese habido una imprenta en la pequeña Huete. ${ }^{12}$ Bartolomé José Gallardo en 1889 sólo se preguntaba si "Castro" podía haber sido el impresor pero no llegaba a dar una respuesta. ${ }^{13}$ Pérez Pastor, citando siempre la edición fechada en 1484, indicaba esta fecha como en la que el "original se concluyó en la ciudad de Huete" y a Juan Vázquez como a su impresor. ${ }^{14}$ Konrad Haebler, en 1896, retomando las afirmaciones de sus predecesores, dudaba también que hubiese existido un impresor en Huete en $1484 .{ }^{15}$

El descubrimiento de la segunda impresión de las Ordenanzas Reales de Díaz de Montalvo, la fechada el 23 de agosto de 1485, cambió radicalmente la escena y con ella las consideraciones que sobre la de 1484 se habían hecho hasta ese momento. Aunque W. A. Copinger y Robert Proctor sólo se limitaron a señalarla como impresa en Huete, ${ }^{16}$ Haebler, en el primer volumen de su Bibliografia Ibérica de 1903, aprovechaba este segundo colofón para revisar su teoría acerca de la edición fechada en 1484 y de la identidad de "Castro". Haebler opinaba entonces

9 Díaz de Montalvo, Ordenanzas Reales, [Huete]: Castro, [11 de noviembre de 1484], hoja [262] r. Biblioteca Nacional de Madrid, Sign. INC. 1338. En la transcripción hemos desarrollado las abreviaturas, normalizado mayúsculas y grafías especiales de la época, y omitido errores tipográficos y signos especiales de puntuación.

10 Díaz de Montalvo, Ordenanzas Reales, [Huete]: Castro, [23 de agosto de 1485], hoja [314] v. Biblioteca Nacional de Madrid, Sign. INC. 1142.

11 Méndez, 1796 (1861): 294-295.

12 Caballero, 1873: 154.

13 Gallardo, 1863-1889: IV, n. 4402.

14 Pérez Pastor, 1887 (1984): 5-6.

15 Haebler, 1896-1897: 81-82.

16 Copinger, 1895-1902: I, 431, n. 4346; y Proctor, 1898-1906 (2002): I, 710, n. 9603. 
que la existencia de dos fechas diversas al final de cada una de las dos impresiones de las Ordenanzas - 11 de noviembre de 1484 y 23 de agosto de 1485-, sólo podían referirse al día de su impresión y no al de su escritura, "Huepte" sólo podía ser el lugar donde ésta se había realizado, y "Castro" indicaba sin duda el nombre de su impresor.

Para demostrar sus afirmaciones y la existencia de un impresor llamado "Castro" en Castilla en 1484 y 1485, Haebler hechaba mano de una simple homonimia y acudía al famoso acuerdo dado a conocer algunos años antes por Antonio López Ferreiro, identificando el "Castro" de las Ordenanzas Reales de Díaz de Montalvo, con el Álvaro de Castro, vecino de Villasandino, que se presenta en 1483 con Juan de Bobadilla ante el cabildo compostelano para imprimir cien breviarios para la diócesis.

No me he decidido ligeramente a reivindicar para la villa de Huete la existencia de una imprenta en el siglo $\mathrm{XV}$, que en escritos anteriores había combatido con razones que aún hoy no parecen extraviadas. Pero lo que a mi parecer resuelve cuestión tan debatida es la existencia de un impresor del apellido Castro entre los del siglo XV y la comparación de los colofones [...]. La segunda edición de Huete tiene el colofón casi de verbo ad verbum concordante con la primera, pero dice: acabose de escreuir en la çibdad de Huepte a veinte y tres dias del mes de Agosto. de mill y quatro cientos y ochenta y cinco años. Ahora bien, si la indicación de la fecha de Huete fuera la de la conclusión del manuscrito no hubiera podido mudarse en esta segunda edición [...]. En vista de todo esto no se puede afirmar que la fecha de Huete sea la de la composición, sino que se debe asegurar que es un verdadero colofón de libro impreso, y desde que sabemos por documentos fehacientes que ya en el año 1483 hubo un maestro de libros de molde, Alvaro de Castro, quien se obligó juntamente con Juan de Bobadilla a imprimir un Breviario de Santiago de Compostela, no hallamos dificultad en suponer que este mismo impresor haya podido trasladarse desde esa villa a la de Huete para componer allí dos ediciones de las Ordenanzas Reales. Sobre sus destinos ulteriores no sabemos más sino que es el autor de algunas bulas de indulgencias; pero de estas no se deduce donde hayan sido ejecutadas. ${ }^{17}$

La posterior aparición de una impresión de las Constituciones Sinodales de Cuenca promulgadas en 1484 y firmadas también "Castro", confirmaban el acierto de Haebler al señalar la existencia de un impresor de este nombre y permitía adju-

17 Haebler, 1903 (1992): 98-99. 
dicar a su taller una serie de bulas sin pie de imprenta hoy atribuidas a su labor en Toledo. ${ }^{18}$ Dicho acierto, sin embargo, llevó a toda la crítica posterior a aceptar también sin reservas las otras dos afirmaciones que Haebler había hecho, interpretando las dos fechas de las Ordenanzas Reales como las de su impresión y, lo que es más importante en este caso, identificando el "Castro" de Huete con el Álvaro de Castro vecino de Villasandino.

\section{2. “CASTRO” VERSUS ÁLVARO DE CASTRO}

La historia que Haebler trazó de "Castro" deja, en palabras de George D. Painter y Leslie A. Sheppard, "a little room for doubt", y no es nuestra intención ponerla aquí en duda, pero su identificación con el Álvaro de Castro que aparece en Santiago el 17 de junio de 1483 no es para nosotros tan evidente como Haebler creyó demostrar. Dicha identificación sirvió a Haebler en 1903 para apoyar documentalmente la existencia de un impresor llamado Castro, hecho que hasta ese momento había sido puesto en duda, y al mismo tiempo confirmar con sus trabajos de impresión la labor de un Álvaro de Castro del que nada se sabía. Hoy, sin embargo, existen en nuestra opinión numerosos datos que parecen contradecir tal identificación.

Si tomamos las localidades en las que trabaja "Castro", su cronología y las impresiones a él atribuidas, y las confrontamos con la información que sobre Álvaro de Castro se menciona en el documento fechado el 17 de junio de 1483 y los datos que se conocen del Breviario compostelano, se presentan ante nosotros una serie de datos difíciles de hilar tanto geográfica como cronológicamente. En 1483 Álvaro de Castro parece trabajar como socio de imprenta de Juan de Bobadilla en Burgos y dice ser vecino de Villasandino, localidad próxima a esa ciudad. Ese mismo año, "Castro" trabaja en el monasterio de San Pedro Martir en Toledo realizando diferentes bulas. En 1484 los ciento veinte Breviarios compostelanos se supone que todavía se estarían llevando a cabo, ya que Juan de Bobadilla y Álvaro de Castro se habían comprometido a entregarlos en el plazo máximo de un año. "Castro", en cambio, aprovechando su inactividad como impresor de bulas en Toledo, pasa a trabajar a Huete, donde el 11 de noviembre de 1484 termina de imprimir las Ordenanzas Reales de Díaz de Montalvo y, en torno a esas mismas fechas, las Constituciones de Cuenca a las que hacíamos referencia más arriba. ${ }^{19}$ En 1987 Antonio Odriozola identifica como pertenecientes al Breviario compostelano encargado en 1483 a Juan de Bobadilla y Álvaro de Castro, algunas hojas en pergamino conservadas en el Archivo del Colegio Notarial de A Coruña, cuya tipogra-

18 Véase Vindel, 1945-1954: VI, 149-151 y 173-179; VIII, 389; Catalogue, 1971: 1xviiilxix y 73-74; y Martín Abad, 2003: 60-61.

19 Sobre estos datos véase Odriozola, 1987; Catalogue, 1971: lxviii-lxix; y Martín Abad, 2003: 60,71 у 79 . 
fía, lejos de confirmar la labor del "Castro" de las Ordenanzas, atribuye su realización al impresor Antonio de Centenera en Zamora. ${ }^{20}$

\section{UNA NUEVA HISTORIA PARA JUAN DE BOBADILLA Y ÁL- VARO DE CASTRO}

Dado que cuanto más se sabe del "Castro" de las Ordenanzas Reales y de su labor en Toledo y Huete, más se aleja éste de Santiago, Burgos y Villasandino, lo más prudente parece ser limitarse, como con Juan de Bobadilla, a los datos que se incluyen en el único documento que los menciona, renunciando a la identificación que Haebler hizo de "Castro" en 1903, y que aún sigue creando más de una confusión. $^{21}$

Pero incluso ateniéndose al acuerdo del 17 de junio de 1483 de Juan de Bobadilla y Álvaro de Castro con el cabildo compostelano, o al menos a la interpretación que de él se ha hecho, observamos que no cesan tampoco aquí los problemas. Con lo que en estos momentos se conoce de la imprenta incunable es difícil aceptar la labor en Burgos del supuesto taller de imprenta de Juan de Bobadilla y Álvaro de Castro, o entender su relación con el impresor Antonio de Centenera que acabaría imprimiendo los cien Breviarios compostelanos en Zamora. George D. Painter y Leslie A. Sheppard ya señalaban en 1971 la imposibilidad de confirmar el trabajo de Juan de Bobadilla en Burgos a pesar de aparecer como vecino de dicha ciudad ante el cabildo de Santiago; y Julián Martín Abad, aun sin dudar de su vecindad burgalesa, sospecha que no trabajó allí, dadas las dificultades en hacer coincidir su labor Burgos con el trabajo en esa misma ciudad del impresor Fadrique de Basilea:

The only other known printer [además de Fadrique de Basilea] who may perhaps have worked at Burgos about this time is Juan de Bobadilla who in 1483 agreed with the Chapter of Santiago de Compostela to print a breviary [...].

Juan de Bobadilla is named as of Burgos ("vezino de Burgos") but is not certainly known to have printed in that town. ${ }^{22}$

20 Véase Odriozola, 1987.

21 George D. Painter y Leslie A. Sheppard, y después Julián Martín Abad, ubican Villasandino en Galicia, indican a Álvaro de Castro como natural de dicho lugar y lo situan en 1483 como vecino de Burgos (Catalogue, 1971: lxviii; y Martín Abad, 2003: 60). El Incunabula Short Title Catalogue pone las dos impresiones de "Castro" de las Ordenanzas Reales bajo el nombre de "Álvaro de Castro", sin corchetes, ni ninguna otra indicación de que se trata de una suposición, a pesar de que en ninguna de las dos impresiones aparezca tal nombre (Incunabula Short Title Catalogue $<\mathrm{http}$ //istc.bl.uk/search/search.html?operation= record\&rsid=292537\&q=1> y <http://istc.bl.uk/search/search.html?operation=record\&rsid= $292537 \& \mathrm{q}=2>$ [consultado el 25 de enero de 2010]).

22 Catalogue, 1971: 1xii. 
¿Podía temer nuestro impresor [Fadrique de Basilea] la competencia de un parigual en la ciudad? La pregunta no está de más puesto que el tesorero García Ruiz de la Mota, antes recordado, se compromete, cuando hace en 1482 el encargo relatado al maestro Fadrique, a reservarle este tipo de encargos y otros de mayor envergadura frente a cualquier otro impresor. ¿Tendría la competencia en Juan de Bobadilla y Álvaro de Castro, natural(sic) de Villasandino, declarándose ambos(sic) vecinos de Burgos que, en 1483, encontrándose en Santiago de Compostela, conciertan con el cabildo la impresión de un Breviarium Compostellanum, trabajo realizado presumiblemente en Zamora y concluido al año siguiente? De momento, parece, pues, que ese compromiso de reservar al impresor los encargos no nos debe hacer pensar en una competencia real e inmediata. ${ }^{23}$

Si volvemos en cambio al documento dado a conocer por López Ferreiro en 1883 transcrito al principio de este artículo, observamos que en él se nos dice únicamente cómo el cabildo compostelano decide el 17 de junio de 1483 encargar a Juan de Bobadilla, vecino de Burgos, y a Álvaro de Castro, vecino de Villasandino, ambos "maestros de hacer breviarios y escrituras de molde", la impresión, encuadernación e iluminación de ciento veinte breviarios en papel. Para nosotros, ni estos datos, ni los aportados por las hojas identificadas por Odriozola en el Archivo del Colegio Notarial de A Coruña, permiten afirmar que dicho acuerdo llegase a ser efectivo, la participación de Juan de Bobadilla y Álvaro de Castro en la impresión de los Breviarios compostelanos, y ni siquiera la existencia de dichos impresores.

Hoy, con el único documento que menciona a Juan de Bobadilla y Álvaro de Castro con el ambiguo "maestros de hacer breviarios y escrituras de molde", y con la ayuda de algunos otros datos documentales, se podría construir una historia totalmente diferente pero tan coherente como en su día fue la de Haebler. Podríamos interpretar el apelativo de "maestros de hacer breviarios y escrituras de molde", no como sinónimo de impresor, como se ha venido haciendo, sino tan sólo para indicar que Juan de Bobadilla y Álvaro de Castro se presentaban ante el cabildo compostelano como capaces de proveerlo con breviarios impresos. Como a Haebler, una sencilla homonimia y coincidencia cronológica nos permitiría identificar a Álvaro de Castro, no con el "Castro" de las Ordenanzas, sino con el mercader Diego Álvarez de Castro, vecino de Burgos en 1482 -quizás en 1483 lo era de Villasandino-; y a su socio con el Juan de Bobadilla que aparece en Burgos en 1486, o incluso con el Juan de Burgos, iluminador de libros, que desarrolla su oficio en esta ciudad al final del siglo XV ${ }^{24}$ Podríamos imaginar un contrato hoy

23 Martín Abad, 2003: 71.

24 Archivo Histórico de la Catedral de Burgos. LIB 15, folios 39-40; RR 27, folio 146; y 
perdido entre el Cabildo compostelano y Centenera que invalidaba el acuerdo previamente alcanzado con Juan de Bobadilla y Álvaro de Castro, o simplemente el encargo de los dos burgalenses al impresor zamorano. ${ }^{25}$ Por supuesto son sólo suposiciones, pero con el mismo valor documental que las hasta hoy comunmente aceptadas, sin ninguna prueba que las demuestre pero también sin ninguna que las contradiga.

El afán por construir una historia de la imprenta castellana bien trabada ha llevado a interpretar de manera no siempre desinteresada los escasos fragmentos de información con los que se contaba, a aceptar sin reservas la inclusión de Juan de Bobadilla y Álvaro de Castro entre los talleres de imprenta castellanos del siglo XV, y a identificar el "Castro" de las Ordenanzas Reales con el Álvaro de Castro vecino de Villasandino. No se ha querido reconocer, en cambio, lo que hoy, a la luz de los datos expuestos, nos parece evidente, que Juan de Bobadilla y Álvaro e Castro restan un completo misterio.

\section{REFERENCIAS BIBLIOGRÁFICAS}

CABALLERO, Fermín: Conquenses ilustres. Doctor Montalvo, Madrid: Tipografía Nacional de Sordo-mudos y de Ciegos, 1873

CATALOGUE of books printed in the XVth Century now in the British Museum, Part X. Spain. Portugal, introducción de George D. Painter y Leslie A. Sheppard, London: British Museum, 1971

COPINGER, W. A.: Supplement to Hain's Repertorium Bibliographicum or Collections Towards a New Edition of That Work In Two Parts, London: Henry Sotheran and Co., 1895-1902

CUESTA, María Luisa: "La imprenta en Santiago de Compostela", GutenbergJahrbuch, 1932/1932: 202-224

GALLARDO, Bartolomé José: Ensayo de una biblioteca española de libros raros y curiosos, Madrid: Imprenta y Estenotipia de M. Rivadeneyra, 4 volúmenes, 1863-1889

LIB 50 1, folio 546. Documentación consultada en línea en Informatización y catalogación del Archivo Histórico de la Catedral de Burgos. Disponible en "http://www.cajacirculo.com/ es/index.php?PA_ID=480\&cod=1955L\&nombre=Alvarez\%20de\%20Castro,\%20Diego";

"http://www.cajacirculo.com/es/index.php?PA_ID=480\&cod=15849RR\&nombre=Bobadilla, \% 20Juan\%20de";

"http://www.cajacirculo.com/es/index.php?PA_ID=480\&cod=11905L\&nombre=Burgos,\%20Ju an $\% 20$ de $5 "$

25 Antonio Odriozola considera "poco probable que el Cabildo de Santiago hiciese un segundo contrato con Centenera cuando aún estaba corriendo el plazo de un año" concedido a Âlvaro de Castro y Juan de Bobadilla (Odriozola, 1987). Sin embargo, algo semejante ocurriría en 1496, cuando el Arzobispo de Santiago por un lado y su Cabildo por otro contrataban contemporánemante la impresión de un breviario para la diócesis con Juan de Porras y Nicolás de Sajonia. Véase Rial Costas, 2007: 36-37. 
HAEBLER, Konrad: Early Printers of Spain and Portugal, London: The Bibliographical Society, 1896-1897

HAEBLER, Konrad: Bibliografia Ibérica del siglo XV. Enumeración de todos los libros impresos en España y Portugal hasta el año de 1500, La Haya - Leipzig: Martinus Nijhoff Karl W. Hiesemann, 1903 (reimpresión facsimilar, Madrid: Julio Ollero, 1992)

HAEBLER, Konrad: Bibliografia Ibérica del siglo XV o hispánica. Segunda parte, La Haya - Leipzig, 1917 (reimpresión facsimilar, Madrid: Julio Ollero, 1992)

Incunabula Short Title Catalogue [en línea]. Disponible en "http://istc.bl.uk/search/search.html?operation=record\&rsid=292392\&q=0"

[Consulta: 25 enero 2010]

Informatización y catalogación del Archivo Histórico de la Catedral de Burgos [en línea]. Disponible en "http://www.cajacirculo.com/es/index.php?PA_ID=476" [Consulta: 3 noviembre 2009]

LÓPEZ, Atanasio (O.F.M.): "La imprenta en Galicia en el siglo XV", GutenbergJahrbuch, 1935/1935: 100-111

LÓPEZ FERREIRO, Antonio: Galicia en el último tercio del siglo XV, Santiago de Compostela: Imp. de la Gazeta, 1883 ( $3^{\text {a }}$ edición, Vigo: Faro de Vigo, 1968)

MARTÍN ABAD, Julián: Los primeros tiempos de la imprenta en España (c. 1471-1520), Madrid: Ediciones del Laberinto, 2003

MÉNDEZ, Francisco: Typographia española o historia de la introducción, propagación y progresos del arte de la imprenta en España, Madrid: Imprenta de la Viuda de D. Joachin Ibarra, 2 volúmenes, 1796 ( $2^{\text {a }}$ edición corregida y con adiciones de Dionisio Hidalgo, Madrid: Imprenta de las Escuelas Pías, 1861)

ODRIOZOLA, Antonio: "Aparecen en La Coruña 8 hojas de un desconocido breviario contratado en 1483", La Voz de Galicia (La Coruña), 2 de abril de 1987

ODRIOZOLA, Antonio y BARREIRO, Xosé-Ramón: Historia de la imprenta en Galicia, A Coruña: La Voz de Galicia, 1992

PÉREZ PASTOR, Cristóbal: La imprenta en Toledo. Descripción bibliográfica de las obras impresas en la imperial ciudad desde 1483 hasta nuestros días, Madrid: Imprenta de M. Tello, 1887 (reimpresión facsimilar, Toledo: Diputación Provincial, Instituto Provincial de Investigaciones y Estudios Toledanos, 1984)

PROCTOR, Robert: An Index to the Early Printed Books in the British Museum: from the invention of printing to the year 1500. With notes of those in the Bodleian Library, London, 2 volúmenes, 1898-1906 (edición facsimilar, Mansfield Centre, CT: Martino Publishing, 2002)

REY SOTO, Antonio: La imprenta en Galicia. El libro gótico, Madrid: Estanislao Maestre, 1934 (edición facsimilar, Santiago de Compostela: Xunta de Galicia, Consellería de Cultura e Benestar Social, 1988)

RIAL COSTAS, Benito: Producción y comercio del libro en Santiago de Compostela (1501-1553), Calambur - Consorcio de Santiago, 2007

VINDEL, Francisco: El arte tipográfico en España durante el siglo XV, Madrid: Imprenta Góngora, 8 volúmenes, 1945-1954 